\title{
Age and strain differences in the effect of distribution of practice on maze learning'
}

\author{
James L. MeGaugh, UNIVERSITY OF CALIFORNIA, IRVNE \\ James M. Cole, SAN JOSE STATE COLLEGE
}

\begin{abstract}
Abstraet
Young and old rats of the $\mathrm{S}_{1}$ and $\mathrm{S}_{3}$ strains were trained on a Lashley III maze with $30 \mathrm{sec}$. or $30 \mathrm{~min}$. intertrial intervals. All Ss except the young $\mathrm{S}_{3}$ rats performed better with the longer intertrial interval. The findings are considered in terms of age and strain differences in memory storage processes.
\end{abstract}

\section{Problem:}

Previous investigators (Fehmi \& McGaugh, 1961; McGaugh, Westbrook, \& Burt, 1961; McGaugh, Jennings, \& Thomson, 1962) using 90-day old Ss of $\mathrm{S}_{1}$ and $\mathrm{S}_{3}$ strains (descendents of the Tryon maze-bright and maze-dull rats respectively) found that the $S_{1}$ strain is superior to the $S_{3}$ strain when training trials are relatively massed (30 sec. intertrial interval), but that the performance of the $S_{3}$ strain is equivalent to that of the $\mathrm{S}_{1}$ strain when a longer interval $(5 \mathrm{~min}$. or more) is allowed between trials.

These results, as well as the finding that the memory of $\mathrm{S}_{1}$ Ss is less adversely affected by post-trial electroconvulsive shock (ECS) (Thomson et al, 1961), have suggested that learning difference may be due to strain differences in memory storage processes. Thompson and his associates (Thompson, 1957; Thompson et al, 1958) have shown that, in comparison with that of adult rats, the memory of young rats is more adversely affected by post-trial ECS, and have interpreted the age difference in ECS effects in terms of possible age differences in memory consolidation processes.

If efficiency of memory storage varies with age, and if the $S_{1}$ and $S_{3}$ maze performance difference under massed practice is due to differences in memory storage processes, then the effect of massed and distributed practice on maze performance should vary with age as well as with strain. The present study was designed to investigate this implication.

\footnotetext{
Subjerets

One hundred and twenty-eight naive descendents from the original Tryon maze-bright $\left(\mathrm{S}_{1}\right)$ and maze-dull $\left(\mathrm{S}_{3}\right)$ rat strains were used as Ss. One half of these Ss (32 males, 32 females) were from 29 to 33 days of age and half ( 32 males, 32 females) were from 142 to 164 days of age at the beginning of pretraining.

Appararus

A 39 in pretraining straight runway and a 4-unit, 8-cul Lashley III alley-maze were used. Each maze alley was 4 in wide, 5 in high, and 47 in long. The runway and the maze were painted medium gray and were covered with hardware cloth. The floor of the maze was marked with white lines 2 in in from both sides of each choice point. Proced ure

The Ss were pretrained for eight days. They were first deprived of food for $24 \mathrm{hr}$., weighed, and given brief exposures to a wet mash food in a goal box on two successive days. This was followed by pre-
}

training trials in the straight alley given over a period of five days. The Ss were then randomly assigned ( $N=8$ per sub-group) to the two practice conditions within age, sex, and strain groups. All of the Ss received five training trials daily for three successive days in the Lashley III maze. The intertrial interval was $30 \mathrm{sec}$. for the massed practice condition and $30 \mathrm{~min}$. for the distributed practice condition. The adult rats were maintained at 85 to $90 \%$ of their first day's weight, but as the young rats could not survive under a rigorous deprivation schedule their diet was supplemented with a daily 5-gm food pellet. All Ss received a wet mash food in the goal box following each pretraining and training trial. An error was recorded if a rat's head and shoulders crossed the white lines in front of blind alleys. Both initial and retracing errors were included.

\section{Results}

The means and standard deviations of total errors made on trials 2 through 15 (excluding the initial exploratory trial) are shown on Table 1. An analysis of variance of the error scores indicated a significant intertrial interval effect $(p<.005 ; F=39.12 ; d f=1 / 112)$, and a significant strain effect $(p<.025 ; F=6.40$; $\mathrm{df}=1 / 112)$. In addition, the interaction $\mathrm{F}$ ratios indicated that the effect of age depended upon sex and strain $(\mathrm{p}<.05 ; \mathrm{F}=4.58 ; \mathrm{df}=1 / 112)$ as well as strain and condition of practice $(p<.025 ; F=5.74 ; d f=1 / 112)$.

The significance of the difference between the subgroup means shown in Table 1 was tested (alpha $=.05$ ) with the Duncan Multiple Range Test (Edwards, 1960, pp. 136-140). None of the differences between males and females within the same age, strain, and intertrial interval sub-groups reached significance. Thus, the sex by strain by age interaction was due to variations in sex sub-groups means rather than to any large sex differences within any particular age, strain, or practice condition.

The means of the young $\mathrm{S}_{1}$ Ss trained under distributed practice were significantly lower $(p<005)$ than those trained under massed practice. The young $\mathrm{S}_{3} \mathrm{Ss}_{\text {, how- }}$ ever, performed equally poorly with both intertrial intervals.

For the older male and female $S_{3}$ and female $S_{1} S s$ the means of the distributed practice sub-groups were significantly lower $(p<.05)$ than those of the massed practice groups. The $\mathrm{s}_{1}$ male mean was also lower under distributed practice, but since the males of this strain also performed well under massed practice (the mean of 22.2 was the lowest for any massed practice group) the difference was not significant.

Comparisons of sub-groups trained with massed practice indicated that the older $S_{1}$ male mean was significantly lower $(p<.05)$ than that of the older $\mathrm{S}_{3}$ males. None of the other comparisons was significant. For the 
Table 1. Means and standard deviations of errors made by young and old male and female rats of the $S_{1}$ and $S_{3}$ strains trained with $30 \mathrm{sec}$. or $30 \mathrm{~min}$. intertrial intervals.

\begin{tabular}{|c|c|c|c|c|c|c|c|c|c|}
\hline & & & & Inte & rtrial In & & & & \\
\hline & & & $30 \mathrm{Secor}$ & & & & Minut & & \\
\hline Strain & & & $\mathrm{s}_{1}$ & $\mathrm{~S}$ & & & $\mathrm{~s}_{1}$ & & $\mathrm{~s}_{3}$ \\
\hline Sex & & M & $\mathrm{F}$ & M & $\mathrm{F}$ & $\mathrm{M}$ & $\mathrm{F}$ & M & F \\
\hline Age & & & & & & & & & \\
\hline & $M$ & 29.5 & 31.1 & 23.3 & 29.9 & 11.1 & 14.9 & 21.6 & 29.6 \\
\hline 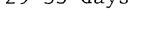 & SD & $(18.0)$ & $(14.6)$ & $(9.1)$ & $(11.5)$ & $(4.3)$ & $(5.9)$ & $(14.4)$ & $(15.5)$ \\
\hline .16 & M & 22.1 & 28.9 & 38.4 & 28.4 & 9.4 & 11.4 & 18.5 & 10.9 \\
\hline & SD & $(9.0)$ & $(14.4)$ & $(7.5)$ & $(13.6)$ & $(5.3)$ & $(4.6)$ & $(8.3)$ & $(6.1)$ \\
\hline
\end{tabular}

distributed sub-groups the means of the older $S_{1}$ and $\mathrm{S}_{3}$ Ss were similar. By contrast, for the younger Ss trained under distributed practice the means of the $S_{1}$ male and female sub-groups were significantly lower $(p<.05)$ than the $S_{3}$ female mean, but not quite significantly lower than the $\mathrm{S}_{3}$ male sub-group mean.

\section{Diseussion}

The results of this study indicate that: (a) with distributed practice, young $s_{1}$ rats are superior to young $\mathrm{S}_{3}$ rats in maze performance; (b) no strain differences in learning were observed with young $s_{1}$ and $s_{3}$ rats trained with massed practice; (c) distribution of practice facilitated the maze performances of older rats from both strains. Thus, the results provide clear indication that the effects of distribution of practice vary with both strain and age. The inferior performance of young $\mathrm{S}_{3} \mathrm{Ss}$ under distributed practice provides further evidence that the difference in maze performance of $S_{1}$ and $S_{3}$ rats is based on differences in processes underlying learning. Comparable findings of age differences in the effect of distribution of practice in Long-Evans rats was reported recently by Doty \& Doty (1964). One plausible interpretation of these results is that the differential effects of distribution of practice are due at least in part to age and strain differences in memory storage efficiency. This interpretation is consistent with other evidence that the degree of retrograde amnesia produced by post-trial ECS or drug treatment is greater in very young rats or very old rats than in rats of intermediate ages (Thompson, 1957; Thompson et al, 1958; Doty \& Doty, 1964).

The data do not fit consistently with any other obvious interpretation. For example, detailed analyses of weight losses and running speeds indicated that the results cannot be readily interpreted in terms of motivational variables.

The interpretation that post-trial ECS and drug effects on memory as well as distribution of practice effects on learning are all based on a single time dependent process underlying memory storage is both parsimonious and appealing. However, since ECS has been shown to produce retrograde amnesia when administered shortly after Ss have learned a discrimination problem to a criterion of 9 out of 10 correct responses it is clear that trial-to-trial improvement cannot be based solely on permanent memory storage (Deutsch, 1962). It appears likely that trial-to-trial improvement found with massed trials given on a single day is based on temporary memory processes and that careful analyses of distribution of practice effects may provide some evidence concerning the nature of temporary storage processes as well as the relationship between temporary and long term storage processes.

\section{References}

DEUTSCH, J. A. The physiological basis of memory. Annu. Rev. Psychol., 1962, 24, 259-286.

DOTY, B. A., \& DOTY, L. A. Effect of age and chlorpromazine on memory consolidation. J. comp. physiol. Psychol., 1964, 57, 331-334.

EDWARDS, A. L. Experimental design in psychological research. New York: Rinehart, 1960.

FEHMI, L., \& McGAUGH, J. L. Discrimination learning by descendents of Tryon maze-bright and maze-dull strains. Psychol. Rep., 1961, 8, 122.

McGAUGH, J. L., JENNINGS, R.D., \& THOMSON, C. W. The effect of distribution of practice on the maze learning of descendents of the Tryon maze-bright and maze-dull strains. Psychol. Rep., 1962., $10,147-150$.

McGAUGH, J. L。, WESTBROOK, W.H., \& BURT, G. Strain differences in the facilitative effects of 5-7-Diphenyl-1-3-Diazadamantan-6-01 (1757 I.S.) on maze learning. J. comp. physiol. Psychol., 1961, 54, 502-505.

McGAUGH, J. L., WESTBROOK, W. H., \& THOMSON, C. W. Facilitation of maze learning with post-trial injections of 5-7-Diphenyl-13-Diazadamantan-6-OL (1757 I.S.). J. comp. physiol. Psychol., 1962, 55, 710-713.

THOMSON, C.W., McGAUGH, J.L., SMITH, C.E., HUDSPETH, W. J., \& WESTBROOK, W. H. Strain differences in the retroactive effects of electroconvulsive shock on maze learning. Canad. J. Psychol., $1961,15,69-74$.

THOMPSON, R., HARAVEY, F., PENNINGTON, D. F., SMITH, J. JR., GANNON, D., \& STOCKWELL, F. An analysis of the differential effects of ECS on memory in young and adult rats. Canad. J. Psychol., 1958, 12, 83-96.

Note

1. This research was supported by Research Grants MY 3541 and MH 10261 from the National Institute of Mental Health, National Institutes of Health, United States Public Health Service. 